\title{
The Objects Detection Increasing Probability Method on Integrated Images of the Sight Surface in Difficult Observation Conditions
}

\author{
Volodymyr Tarshyn ${ }^{1}$, Alexander Tantsiura ${ }^{2}$, Yaroslav Kozhushko ${ }^{3}$, Volodymyr Vasylyshyn ${ }^{4}$, \\ Viktor Mosharenkov ${ }^{5}$, Yuliia Tarshyna ${ }^{6}$ \\ ${ }^{1}$ Doctor of Technical Sciences, Associated Professor, Head of the Department of Armament of Radio \\ Engineering Troops, Kharkiv National University named after Ivan Kozhedub Air Force, Ukraine, vratar-73@ukr.net \\ ${ }^{2} \mathrm{PhD}$, Researcher, Department of Air Force Research Center, Kharkiv National University named after Ivan \\ Kozhedub Air Force, Ukraine, shurik.lucky@ gmail.com \\ ${ }^{3} \mathrm{PhD}$, Senior Researcher, Department of Air Force Research Center, Kharkiv National University named after Ivan \\ Kozhedub Air Force, Ukraine, oficer2003@ukr.net \\ ${ }^{4}$ Doctor of Technical Sciences, Associated Professor, Head of the Department of Radio Electronic Systems of \\ Air Force Control Points, Kharkiv National University named after Ivan Kozhedub Air Force, Ukraine, \\ vladvas@ukr.net \\ ${ }^{5} \mathrm{PhD}$, Senior Lecturer, Department of Air Force Research Center, Kharkiv National University named after Ivan \\ Kozhedub Air Force, Ukraine, mosharenkov@ gmail.com \\ ${ }^{6}$ Student, National Technical University "Kharkiv Polytechnic Institute", lilmayble@ gmail.com
}

\begin{abstract}
The paper develops and studies the objects detection increasing probability method on integrated images of the sight surface (SS) in difficult observation conditions. The objects detection increasing probability in integrated television (TV) and infrared (IR) images is achieved by taking local brightness and contrast properties of the image sections into account. The objects detection probability increasing method is proposed on integrated images of VS obtained under difficult environmental conditions. As a possible option of the method practical implementation, an algorithm is proposed for integrating TV and IR images, taking into account their structural similarity.
\end{abstract}

Key words: Vision surface image, detection probability, integration method, multi spectral images

\section{INTRODUCTION}

Ensuring the required value of the objects correct detection (localization) probability on SS images is one of the key tasks that is solved in optoelectronic correlation-extreme navigation systems for unmanned aerial vehicles (UAVs), observation and guidance systems [1]. Most modern multispectral optoelectronic visual observation systems incorporate the TV and IR range sensors that provide high-quality SS images in accordance with the observation conditions [2].

At the same time, the ability to provide the desired probability of the objects correct detection substantially depends on the complexity of observation conditions, which are caused by the presence of atmospheric formations, precipitation, active and passive interference, camouflage and imitation tools, shadows from neighboring objects, etc. [3]. In general use of the
TV and IR information acquisition sensors during difficult surveillance conditions the ability to detect and identify targets (objects) by the surveillance system improves. However, the best result can only be achieved with the integrated use of information from the different spectral range sensors through the use of methods for the combining multispectral images [4].

\subsection{Problem analysis}

The application of the known methods for combining multispectral images makes it possible to reduce the influence of complex observation conditions on the probability of the objects correct detection on the SS images by combining the distinctive features of the original images in different spectral ranges [3], [5]. At the same time, the use of traditional methods for combining multispectral images as a tool to ensure the necessary probability of the objects correct detection in difficult observation conditions, does not always allow to achieve the desired result due to the fact that the informative differences of the original images are not used in full capacity [5]-[6]. The application assessment results on a set of known methods for integrated images are obtained in [7]. In general use, the application of modern integrated methods is to find the functions of spatial transformation and brightness conversion of the original images.

Spatial transformation is usually implemented by the widely known methods, for example, using control points or polynomial, sometimes affine transformation, which are widely considered in popular publications [8]-[9]. Frequently used methods for converting brightness that satisfy the time requirements for the process of forming an integrated image of the sight surface are disclosed in [10]. 
Finding a brightness conversion function is to apply one or more operators to all discrete samples of the output digital images of the sight surface.

In work [7], it was determined that the method of integrated images using weight coefficients is rather simple to implement and the best criterion for the maximum signal-to-noise ratio:

$$
F_{k}(i, j)=\alpha F_{t v}(i, j)+(1-\alpha) F_{i r}(i, j)
$$

where $F_{k}$ - is the integrated image element; $\alpha$ weight coefficient; $F_{t v}(i, j), F_{i r}(i, j)$ - are the source images in the TV and IR range, respectively.

The weight coefficient is calculated by the following expression [6]:

$$
\alpha=\frac{\sum_{0}^{\mathrm{M}-1} \sum_{0}^{\mathrm{N}-1} \mathrm{~F}_{\mathrm{ir}}(\mathrm{i}, \mathrm{j}) / \mathrm{M} \cdot \mathrm{N}}{\sum_{0}^{\mathrm{M}-1} \sum_{0}^{\mathrm{N}-1} \mathrm{~F}_{\mathrm{ir}}(\mathrm{i}, \mathrm{j}) / \mathrm{M} \cdot \mathrm{N}+\sum_{0}^{\mathrm{M}-1} \sum_{0}^{\mathrm{N}-1} \mathrm{~F}_{\mathrm{tv}}(\mathrm{i}, \mathrm{j}) / \mathrm{M} \cdot \mathrm{N}},(2)
$$

where $\mathrm{M}$ and $\mathrm{N}$ the rows and the columns number of the source images, respectively.

At the same time, the coefficient determination in expression (2) provides for an increase in the noise component, which makes it impossible to take into account local features of objects, backgrounds, and interference. In this case, the brightness, contrast and structural features of the original images are not taken into account.

The aim of the paper is to present and evaluate the effectiveness of the probability increasing method for the objects' detection on integrated SS images in difficult observation conditions by preliminary determining the properties of the informative areas of the source images during integration.

\section{MAIN MATERIAL}

\subsection{The objects detection increasing probability method on integrated images of the sight surface.}

For a preliminary determination of the source images informative areas properties during their integration, it is necessary to determine these areas. The determination of the source images informative areas is carried out by determining the measure of the structural images' similarity. Studies conducted in [11] showed that the magnitude of the structural similarity measure is directly related to the local features of the SS location. At the same time, such an indicator when solving problems of the correct detection increased probability of the observation objects has not been considered before. In future, the term 'integration' is applied in the context of finding the brightness conversion of the source images, since in some cases (depending on the optical design of the optoelectronic observation systems) the spatial conversion stage during integration may be absent

A structural similarity measure is one of the known indicators for evaluating images and includes an assessment of local differences in brightness $\mathrm{B}_{\mathrm{m}, \mathrm{n}}$, objects contrast $\mathrm{C}_{\mathrm{m}, \mathrm{n}}$ and structure $\mathrm{K}_{\mathrm{m}, \mathrm{n}}$ and the images' backgrounds, and is most often calculated in a sliding window with a size of $\mathrm{m} \times \mathrm{n}[12]-[13]$ :

$$
\|\mathrm{Q}\|=\left\|\mathrm{B}_{\mathrm{m}, \mathrm{n}} \cdot \mathrm{C}_{\mathrm{m}, \mathrm{n}} \cdot \mathrm{K}_{\mathrm{m}, \mathrm{n}}\right\| .
$$

In this case, a matrix of the structural similarity measure values is formed in the range $[0,1]$, which characterizes the images local differences in relation to which the integration is performed:

$\mathrm{Q}_{\mathrm{M}, \mathrm{N}}=\left[\begin{array}{cccc}\mathrm{Q}_{\mathrm{m}, \mathrm{n}} & \mathrm{Q}_{\mathrm{m}, \mathrm{n}+1} & \ldots & \mathrm{Q}_{\mathrm{M}, \mathrm{N}-\mathrm{n}} \\ \mathrm{Q}_{\mathrm{m}+1, \mathrm{n}} & \mathrm{Q}_{\mathrm{m}+1, \mathrm{n}+1} & \ldots & \mathrm{Q}_{\mathrm{m}+1, \mathrm{~N}-\mathrm{n}} \\ \vdots & \vdots & \ldots & \vdots \\ \mathrm{Q}_{\mathrm{M}-\mathrm{m}, \mathrm{n}} & \mathrm{Q}_{\mathrm{M}-\mathrm{m}, \mathrm{n}+1} & \ldots & \mathrm{Q}_{\mathrm{M}-\mathrm{m}, \mathrm{N}-\mathrm{n}}\end{array}\right]$

Each matrix element $\mathrm{Q}_{\mathrm{M}, \mathrm{N}}$ characterizes the local differences in brightness $\left\|\mathrm{B}_{\mathrm{m}, \mathrm{n}}\right\|=\frac{2 \overline{\mathrm{F}_{\mathrm{tv}}} \overline{\mathrm{F}}_{\mathrm{ir}}}{\overline{\mathrm{F}}_{\mathrm{tv}}^{2}+\overline{\mathrm{F}}_{\mathrm{ir}}^{2}}$, contrast $\left\|\mathrm{C}_{\mathrm{m}, \mathrm{n}}\right\|=\frac{2 \sigma_{\mathrm{Ftv} \text { Fir }}}{\sigma_{\text {Fir }}^{2} \sigma_{\text {Fir }}^{2}}$ and structure $\left\|\mathrm{K}_{\mathrm{m}, \mathrm{n}}\right\|=\frac{\sigma_{\mathrm{Ftv} \text { Fir }}}{\sigma_{\text {Fir }} \sigma_{\text {Fir }}}$ of the source (original) images. An example of assessing the structural similarity degree for the TV and IR images of the SS shown in Fig. 1 with a size of $680 \times 680$ pixels obtained from digital aerial photography with the Hasselbald-39 camera and the "Malachite" thermal imager, which operates in the wavelength range of $8-14 \mu \mathrm{m}$ [6], are shown in the matrixes form in Fig. 2.

The images similarity degree in brightness, contrast and structure is characterized by a color change in accordance with local changes $B_{m, n}$ (Fig. 2 a), $C_{m, n}$ (Fig. 2 b), $K_{m, n}$ (Fig. 2 c) and $Q_{m, n}$ (Fig. 2 d) ranging from 0 (blue) to 1 (yellow). Matrixes analysis of the local differences in images (Fig. 2) allows us to determine the differences nature in the original images, as well as to identify areas with the maximum similarity degree. At the same time, there is a need to numerically determine the structural similarity measure values for the different spectral images regions, which significantly affects the integration result. 


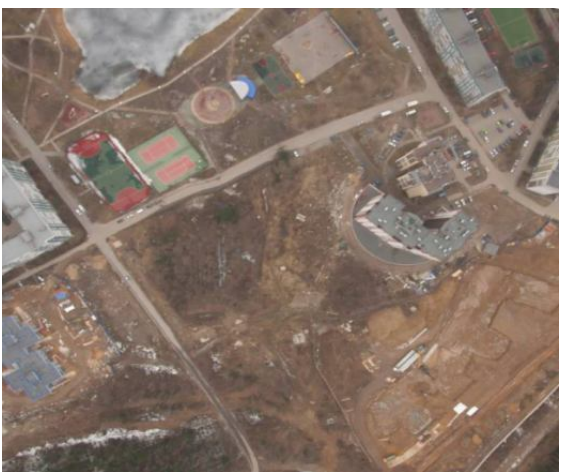

a)

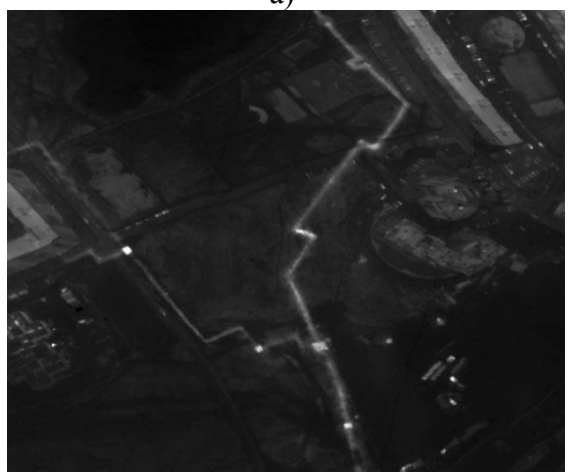

b)

Figure 1: Examples: a) TV images; b) IR images

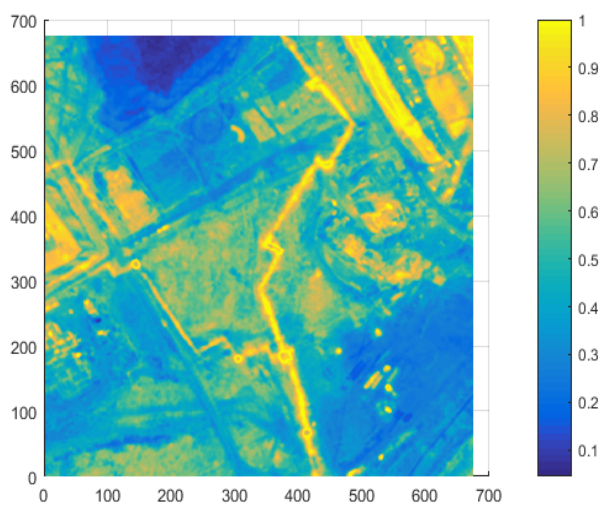

a)

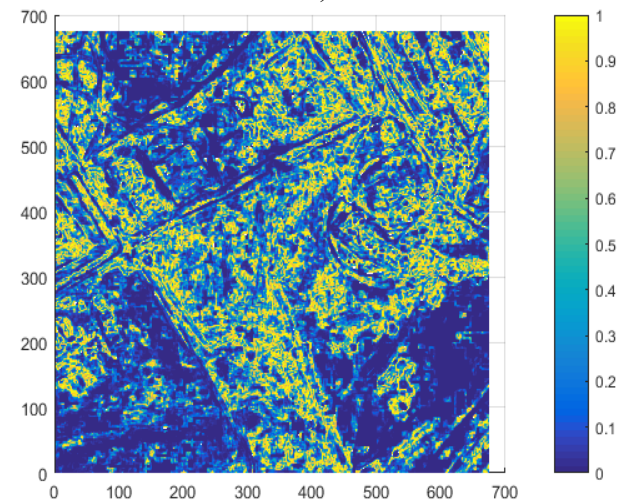

b)

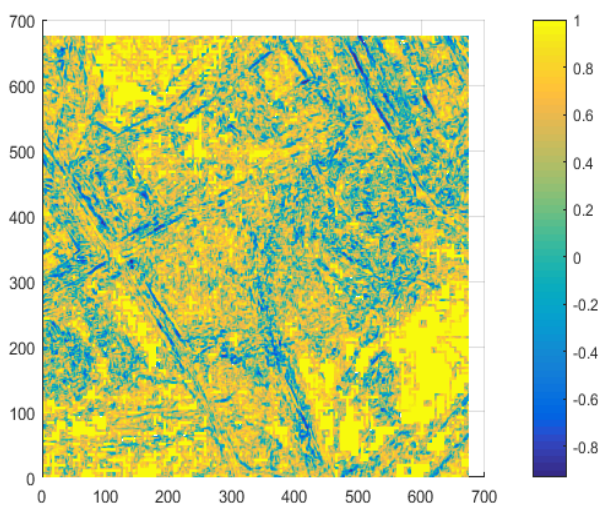

c)

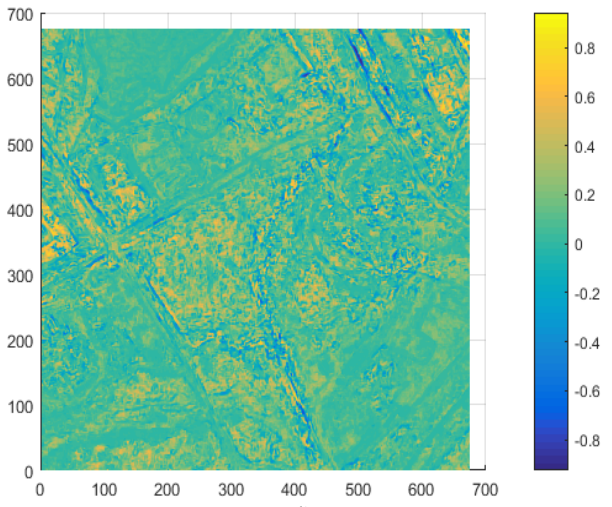

d)

Figure 2: Graphic representation of the local differences matrixes images: a) in brightness; b) in contrast; c) by structure; d) in terms of structural similarity measures

To determine the structural similarity measure effect of the output TV and IR images on the result of integration, a mathematical model was created that implements the integration process by expression (1) where the weight coefficient $\alpha$ is determined in accordance with the known approach (2). As initial sets of pairs of the TV and IR images with different typical object saturation were used [1], [11]. When modeling, the image elements brightness was set in the range from 0 to 255 . In accordance with the developed model, the structural similarity $\mathrm{Q}$ measure was calculated for the source images, then the integration was performed and the value of the normalized correlation coefficient between the source and resulting images $\mathrm{R}$ was defined/

According to the statistical modeling results, the normalized correlation coefficient dependence of the resulting and source images was obtained on the degree of the source images structural similarity, which is shown in Fig. 3. 


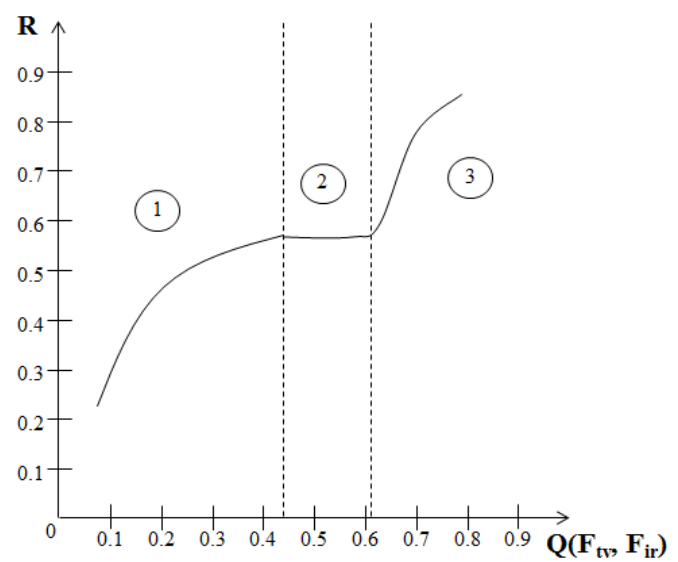

Figure 3: The dependence of the resulting and source images normalized correlation coefficient on the measure of the source images structural similarity.

Based on the statistical modeling, it was found that the image integration result substantially depends on the value of their structural similarity measure. In this case, the influence of the structural similarity measure on the image integration result is characterized by three situations, which are noted in Fig. 3. In accordance with this:

1. With a $Q$ value in the range of $0 \ldots 0.44$, the images have significant differences, therefore, the combination ( integration) of such images causes the appearance of both characteristic features source images on the resulting image; this case is typical for difficult sighting conditions.

2. In a case when $Q$ takes values in the range of $0.45 \ldots$ 0.61 , the original images have slight structural differences. The combination of such images enhances the objects contours in the resulting image along with a decrease in the average brightness level. In this case, in order to increase the signal-to-noise ratio the integration is reasonable, but only if the brightness of the resulting image is increased programmatically.

3 . With a $Q$ value in the range of $0.62 \ldots 1$, the original images are characterized by a high degree of structural similarity. In this case, the integration images result is practically no different from the original images, however, the resulting image has a slightly lower brightness than the average level. Then the integration is unreasonable, and in order to detect the objects in the SS image, you must select one of the source images.

Empirically, the weight coefficient $\alpha$ significance for the areas of the Q characteristic value was determined, which ensures that local differences in the source images are taken into account. The source images areas with a $Q$ value in the range of $0.62 \ldots 1$ should correspond to a coefficient $\alpha=0,55$, in the case when a
$\mathrm{Q}$ adopts values in the range of $0.45 \ldots 0.61$, the weight coefficient should have a value $\alpha=0,4$. In the case when a $Q$ takes on a value in the range $0 \ldots 0.44$, the weighting factor must have a value $\alpha=0,22$.

The developed approach application to determining the weight coefficient used for the integrated images is ambiguous in accordance with the pair of coefficients $\alpha$ and $1-\alpha$ the source (original) image. The solution to this ambiguity depends on the features of the original images' formation, which must be taken into account when combining.

It is known that the image structure in the TV range depends on the spectral density of the observation object energy brightness $L(\lambda)$ and the spectral density of the ideally reflecting reference surface energy brightness $\mathrm{L}_{0}(\lambda)$ under the same conditions and is defined as $\mathrm{r}(\lambda)=\mathrm{L}(\lambda) / \mathrm{L}_{0}(\lambda)$ [3], [5]. Due to the difference in the spectral brightness coefficients of the various objects and backgrounds, the contrast of the objects in a TV image is random. The image structure of the IR range depends on the average thermal contrast over the object area $\overline{\Delta \mathrm{T}_{\mathrm{R}}}$ and the standard deviation of the contrast variations over the object area $\sigma_{\Delta \mathrm{T}}$ and is defined as $\Delta \mathrm{T}_{\mathrm{R}}=\left({\overline{\Delta \mathrm{T}_{\mathrm{R}}}}^{2}+\sigma_{\Delta \mathrm{T}}^{2}\right)^{1 / 2}[3,5]$. The practical use of the IR sensors indicate that in most cases, reconnaissance objects will have a positive contrast relative to the background, and the average brightness level of the entire image is less than the TV image. Thus, it is possible to solve the ambiguity of determining the weight coefficients correspondence with the original image based on the features of the IR and TV images formation. Since the image objects contrast in the IR range relative to the background is positive in most cases, the source image in the IR range corresponds to a coefficient $1-\alpha$, respectively, the TV image corresponds to a weighting factor $\alpha$.

Thus, we propose a method for increasing the likelihood of the observation objects correct detection on the integrated TV images, based on the determining the structural similarity measure between the TV and IR images. The method allows to take into account local differences in the original images and the features of their formation in different spectral ranges. In fig. 4 an example of the developed method practical implementation in the form of an algorithm flowchart is shown that describes the integration process of the multi spectral SS images based on a structural similarity measure. 


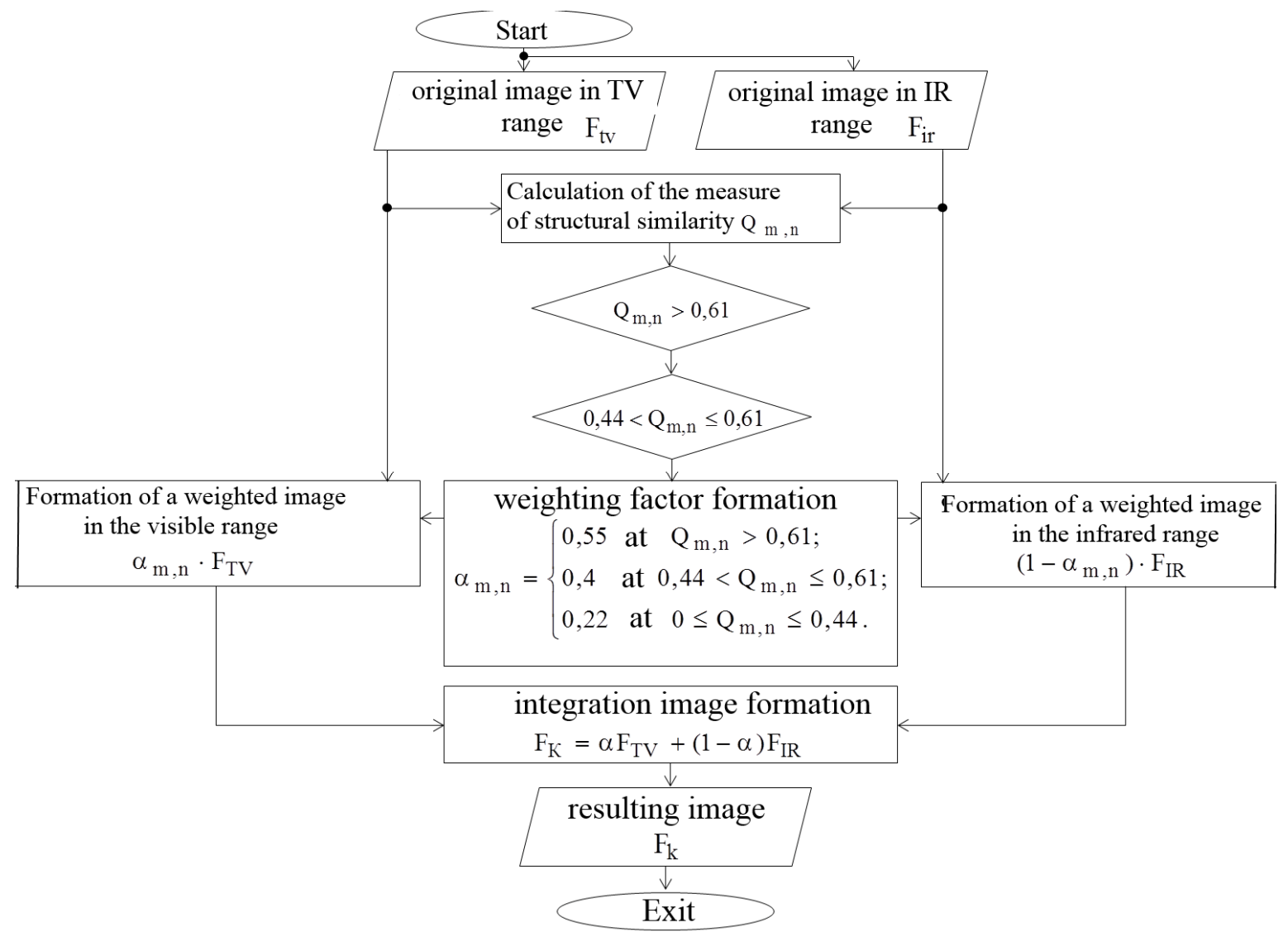

Figure 4: The algorithm block diagram for combining the SS images based on the assessment of the structural similarity degree

\subsection{The results of applying the observation object detection increasing probability method on the integrated SS images.}

In order to evaluate the results of applying the observation object detection increasing probability method on the integrated SS images using a method based on the structural similarity degree assessment, we compared these results with the results obtained for the known methods. A comparative analysis was carried out by calculating the objects detection probability at different signal-to-noise ratios for each of the integrated methods. The correct detection (D) probability and the false alarm (F) was calculated using the expressions presented in [14]-[15]. The segmentation threshold in accordance with the Otsu method was determined, which is based on minimizing the class variance of the image histogram, which is defined as the weighted sum of the two classes variances [16]:

$$
\sigma_{\omega}^{2}=\omega_{1} \sigma_{1}^{2}+\omega_{2} \sigma_{2}^{2}
$$

where $\omega_{1}$ and $\omega_{2}$ there are the first and second classes probabilities.

Minimizing variance within a class is equivalent to maximizing variance between classes:

$$
\sigma_{b}^{2}(t)=\sigma^{2}-\sigma_{\omega}^{2}(t)=\omega_{1}(t) \omega_{2}(t)\left[\mu_{1}(t)-\mu_{2}(t)\right],
$$

where $\mu_{1}, \mu_{2}-$ is the arithmetic mean for each of the classes.

According to the calculated threshold, the image is segmented into an object and background. By statistical modeling, the averaged dependences of the objects detection probability on the integrated SS images based on the signal-to-noise ratio for various methods of the image integration with the probability

of false alarm $\mathrm{F}=1,78 \cdot 10^{-4}$ were obtained. The objects detection was based on the segmentation of the images with low, medium and high object saturation using the Otsu method. The curves were obtained from the 100 noise realizations with a normal distribution law and zero mathematical expectation for each of the methods.

The application effectiveness of the developed method for increasing the probability of the correct detection was assessed and the difference in the probabilities of the developed method correct detection and the method where the resulting image was obtained using the weight coefficient method is shown. In fig. 5 it shows the averaged dependences of the objects detection probability on the integrated SS images of the signalto-noise ratio for various integration methods. 


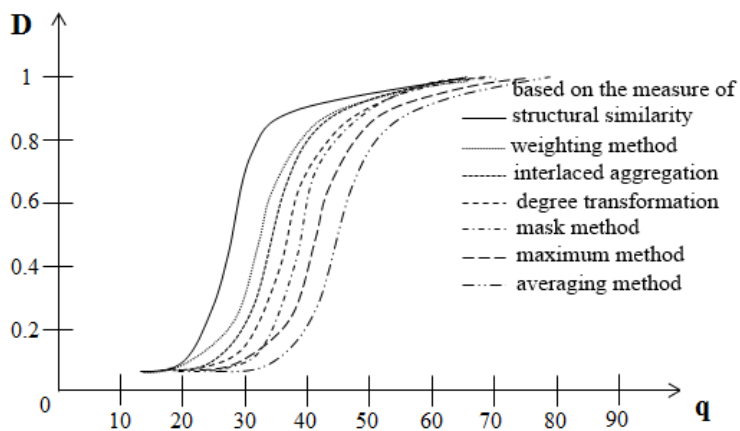

Figure 5: Dependence of the objects detection probability on the integrated SS images obtained by different methods, on the magnitude of the signal-tonoise ratio.

The simulation results show that, with equal signal-tonoise ratios, the best objects' correct detection probability on the integrated images is ensured when integrated by a method based on the structural similarity degree assessment.

For clarity, the presenting the results obtained as an example of the source images, the images selected are shown in Fig. 1. In fig. 6 Integration results of the original images by the weighting coefficients methods (a) and the developed method (b) are shown. The assessing results of the objects' correct detection probability by the TV and IR output, as well as the integrated images by two methods, are shown in Fig. 7. In mathematical modeling, the noise seemed to be a random variable distributed according to the normal law with zero mathematical expectation.

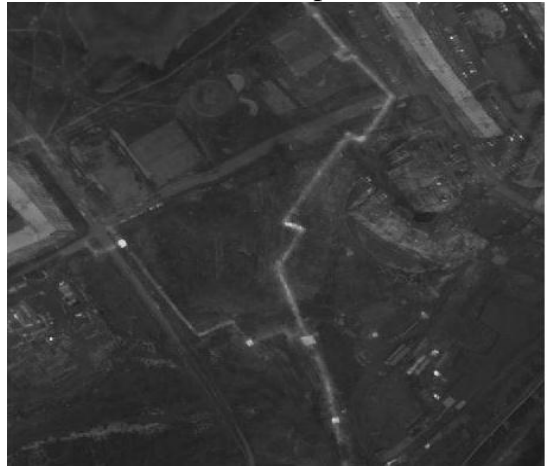

a)

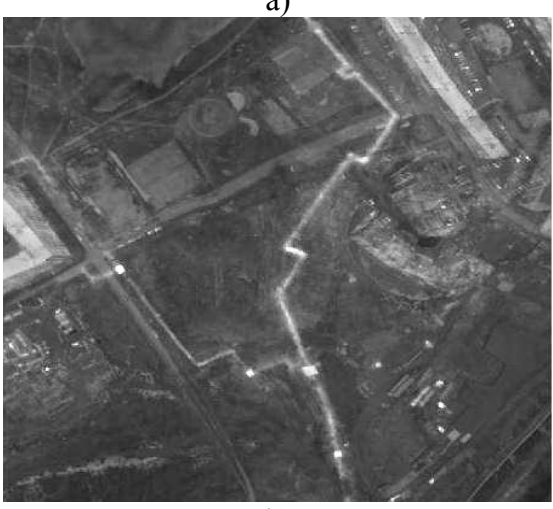

b)

Figure 6: The integrated result of the TV and IR images a) by the weighting coefficients method; b) by the structural similarity assessing method.
From the graph in Fig. 7 it shows that the developed method application to increase the detection probability by integrating the SS images by the method based on a structural similarity measure can significantly increase the probability of detecting an object in an integrated SS image compared to the original images and the images obtained by other integration methods.

The application of the developed method can be useful for the technical sighting (vision) systems (technical surveillance) equipped with multispectral sensors (TV, IR, radar), to improve the detection and the identification of objects in difficult observation conditions.

The mathematical modeling results presented in the work indicate that the developed method allows not only to increase the likelihood of detecting objects on the integrated SS images obtained in the difficult environmental conditions, but it is also adaptive to its changes.

When studying the developed method effectiveness, it wasn't the goal to study its performance and the SS scanning speed effect on the images integration result. This issue requires further study. However, the developed method and the algorithm for the integrated images can very well be implemented in surveillance systems with a low scanning space speed. Such modern technical surveillance systems include the battlefield monitoring means, monitoring compliance with the split (separation) mode in the military conflict situations, and protecting the state border [16]-[17].

\section{CONCLUSION}

A method for increasing the objects detection probability of observation on the integrated SS images obtained in different spectral ranges in difficult environmental conditions is proposed. The presented method is based on the images' integration in the TV and IR ranges, which allows to obtain a resulting image with different areas of the original images captured in it.

Unlike the existing integration methods, where the conversion operator is applied in the same way to all parts of the image, the developed method provides for the weighting coefficients formation taking into account the structural similarity and local features of the original images. The transformation operators' formation of the source images is carried out on the basis of calculating the degree of their structural similarity in a moving (sliding) evaluation window.

Based on the obtained results analysis, it was found that the proposed application approach to the integration of the SS multispectral images makes it possible to significantly increase the signal-to-noise ratio of the integrated image in comparison with the original ones and, as a result, increase the objects' correct detection probability of observation in the integrated SS images up to $25 \%$. 
The proposed method can be used in the optoelectronic surveillance systems, correlation-extreme navigation systems, reconnaissance systems, and other optoelectronic systems critical to the quality of the obtained images under difficult observation conditions.

\section{REFERENCES}

1. V.K. Baklytskyu. Methods for filtering signals in correlation extreme navigation systems, Radio and communication, Moskov, USSR, 1986, $216 \mathrm{p}$.

2. N.Yeromina, A.Sotnikov, V.Tarshyn. Formation of the decisive function of correlation-extreme navigation systems by the criterion of the generalized mutual correlation coefficient, Bulletin of NTU "KhPI", Ukraine, Kharkiv, 2016, №50(1222), pp. 68 - 73.

3. A.Sotnikov, V.Tarshyn, N.Yeromina, S.Petrov, N.Antonenko. A method for localizing a reference object in a current image with several bright objects, Eastern-European Journal of Enterprise Technologies, 2017. Vol. 3. № 9 (87). pp. 68-74.

4. A.M. Sotnikov. Models of current images formed by the channels of the combined correlation-extreme navigation system of an unmanned aerial vehicle, Modern information technologies in the field of security and defense, Ukraine, Kyiv, 2018, pp. 29-38.

5. A.M. Sotnikov. Preparation of reference images for high-speed correlation-extreme navigation systems based on the use of direct correlation analysis, Science and technology of the Air Force of the Armed Forces of Ukraine, Ukraine, Kharkiv, 2015, pp. $69-73$.

6. O.Vorobiov, A.Sotnicov, A.Tantsiura. Models of current images that are formed by the combined correlation-extremal navigation system of a non-flammable aircraft. Modern information technologies in the sphere of security and defence, Ukraine, Kyiv, - 2018. Vol.2, No.32. - pp. 29-36.

7. A.Tantsiura, D.Kolomiets, I.Tabakova, I.Hannoshyna, N.Serdiuk, O.Yelieazarov, T.Voichenko Evaluation of the potential accuracy of correlation extreme navigation systems of low-altitude mobile robots. International Journal of Advanced Trends in Computer Science and Engineering, 8(5), September - October 2019, pp. 2161 - 2166 DOI: $10.30534 /$ ijatcse/2019/47852019
8. N.Yeromina, S.Petrov, A.Tantsiura, M.Iasechko, V.Larin. Formation of reference images and decision function in radiometric correlationextremal navigation systems. Eastern-European Journal of Enterprise Technologies. - 2018. Vol.4, No.9 (94). - pp. 27-35. DOI: 10.15587/1729-4061.2018.139723

9. A.Tantsiura, A.Bondarchuk, O.Ilin, Yu.Melnyk, O.Tkachenko, K.Storchak The image models of combined correlation-extreme navigation system of flying robots. International Journal of Advanced Trends in Computer Science and Engineering, 8(4), July- August 2019, pp. 1012 1019 DOI: $10.30534 /$ ijatcse/2019/05842019

10. E.Kulikov, A.Triphonov. Estimation of parameters of signals against noise. Soviet radio, Moskov, USSR, 1978, $296 \mathrm{p}$.

11. A.V. Mezentsev, S.V. Mironyuk, A.M. Sotnikov [et al.], Methods for the selection of informative image areas based on the theory of fractal analysis, Registration, storage and processing of data, 2015, No. 2, pp. 29-38.

12. Z. Wang, A.C. Bovik, A universal image quality index, Signal processing letters, 2002, No. 5, pp. 1-4

13. G.Piella, H.Heijmans, A new quality metric for image fusion, Image Processing, 2003, No. 2, pp. 173-176.

14. Radio-electronic systems: the fundamentals of construction and theory: reference; under the editorship of J.D. Shirman, Moscow: Radio Engineering, 2007, 512 p.

15. A.I. Strelkov, V.I. Barsov, T.A. Strelkova [et al.], Evaluation of the effectiveness of the method of accumulation of a series of short exposure low-contrast television frames, Collection of scientific works HUPS, 2007, No. 1 (13), pp. 44-47.

16. Hazel Mae A.Adriano, Dustin A.Reyes, Kristelee Jem B.Tan, Daniel D.Zagada, Reggie C.Gustilo, Defensive turret defensive turret with fully automated motion detection using infrared technology, International Journal of Emerging Trends in Engineering Research, 7(9), September 2019, pp. 239-246. https://doi.org/10.30534/ijeter/2019/05792019

17. H. Khudov, I. Khizhnyak, V. Koval, at al., The efficiency estimation method of joint search and detection of objects for surveillance technical systems, International Journal of Emerging Trends in Engineering Research, 8(3), March 2020, pp. 813-819. https://doi.org/10.30534/ijeter/2020/34832020 